\title{
Solar Internal Rotation, the Boundary Layer Dynamo and Latitude Distribution of Activity Belts
}

\author{
G. Belvedere ${ }^{1}$, M.R.E. Proctor $^{2}$ and G. Lanzafame ${ }^{1}$ \\ ${ }^{1}$ Istituto di Astronomia, Università di Catania, Italy \\ ${ }^{2}$ Department of Applied Mathematics and Theoretical Physics, University \\ of Cambridge, England
}

\begin{abstract}
We suggest that the latitude distribution of solar activity belts and the related equatorward or poleward migration of different tracers of the solar cycle are a natural consequence of the internal radial profile of angular velocity via the working of a dynamo in the boundary layer beneath the convection zone. This has been confirmed by the results of a non-linear dynamo model in a very thin spherical shell which show that dynamo action may reasonably take place in the boundary layer and reproduce the observed surface phenomenology.

Extending the argument to late main-sequence stars, it is reasonable to think that observations of the latitude distribution and migration of stellar active regions by current sophisticated techniques may make it possible to infer their internal rotation profile in a simple and direct way.
\end{abstract}

\section{Belts as a consequence of internal rotation}

The location of dynamo action in the boundary layer at the bottom of the convection zone is indirectly supported by the most recent helioseismological results (Belvedere, 1990). Indeed helioseismic data (e.g. Harvey, 1988; Brown et al., 1989; Dziembowski et al. 1989; Libbrecht, 1989) show that the surface differential rotation persists throughout the convection zone $\left(1 R_{\odot} \rightarrow 0.7 R_{\odot}\right)$, where the radial gradient of angular velocity vanishes, while rigid rotation dominates beneath the c.z. $\left(0.65 R_{\odot}\right)$, with angular velocity $\omega_{\circ}=2.7 \cdot 10^{-6} \mathrm{rad} \mathrm{s}^{-1}$, which coincides with the surface value at latitude $\lambda=37^{\circ}$.

In the light of these data, radial shear driven dynamos operating in the c.z. might be unrealistic, whereas a boundary layer dynamo is strongly supported by them, on the basis of the following argument (referring to the northern hemisphere). 
Since considerations of the helicity of convective overshooting motions show that the dynamo parameter $\alpha$ should be negative in the boundary layer (Yoshimura, 1975a; Glatzmaier, 1985a,b), and, interpolating the helioseismological results in the boundary layer itself, the radial angular velocity gradient $\partial \omega / \partial r$ should be positive for $\lambda<37^{\circ}$ and negative for $\lambda>37^{\circ}$, the dynamo criterion (Parker 1955; Yoshimura 1975b), based on the sign of the product $\Gamma=\alpha \partial \omega / \partial r$, allows equatorward propagation of dynamo waves $(\Gamma<0)$ at lower latitudes $\left(\lambda<37^{\circ}\right)$ and poleward propagation at higher latitudes, in agreement with the observational evidence shown by different tracers of the solar cycle.

Indeed spots and most faculae (equatorial activity belt; $\lambda<35^{\circ}-40^{\circ}$ ) do migrate towards the equator during the solar cycle, while polar faculae, filaments and large scale magnetic flux (polar belt; $\lambda>40^{\circ}-50^{\circ}$ ) do evidence poleward migration.

We want to stress here that all this has a profound significance for the interpretation of solar activity global features:

(1) the latitude distribution of solar activity belts and the related migration are a natural and direct consequence of the structure of the velocity field in the solar interior;

(2) dynamo action in the boundary layer can still (and better) account for the observed phenomenological evolution in the course of the solar cycle, and remains the basic mechanism to understand solar (and stellar) magnetic activity.

\section{The working of boundary layer dynamo}

The present picture has been given substantial support by the results of a nonlinear dynamo model in a very thin $\left(0.05 R_{\odot}\right)$ spherical shell, representing the boundary layer, with full time and latitudinal resolution, and an integrated representation only in the radial direction (the so-called radial truncation).

The mean field dynamo equations for the time evolution of the magnetic field $\boldsymbol{B}=\boldsymbol{B}(r, \theta) \phi+\nabla \times(A(r, \theta) \phi)$, where $r, \theta$ are spherical polar coordinates, $\phi$ is the unit vector in the azimuthal direction, $B \phi$ is the toroidal and $B_{p}=\nabla \times A \phi$ the poloidal part of $B$, are:

$$
\begin{gathered}
\frac{\partial A}{\partial t}=\alpha F(r, \theta) B+\eta_{T}\left[\nabla^{2}-\frac{1}{r^{2} \sin ^{2} \theta}\right] A, \\
\frac{\partial B}{\partial t}=r \sin \theta B_{p} \cdot \nabla\left[\frac{U(r, \theta)}{r \sin \theta} \phi\right]+\eta_{T}\left[\nabla^{2}-\frac{1}{r^{2} \sin ^{2} \theta}\right] B .
\end{gathered}
$$

Here $\alpha F$ is the usual $\alpha$-effect, with $F$ representing its spatial structure and $\alpha$ its magnitude. The quantity $\eta_{T}$ is a turbulent diffusivity.

The dynamical influence of the magnetic field enters the model through its effect on the differential rotation $U(r, \theta)=u_{\circ}+u$, where $u_{0}$ is a prescribed velocity field and $u$ is a perturbation driven by the mean Lorentz force and subject to viscous damping.

The simplest equation that encompasses these features is: 


$$
\rho \frac{\partial u}{\partial t}=\frac{1}{\mu_{o}}[(\nabla \times B) \times B]_{\phi}+\rho \nu_{T}\left[\nabla^{2}-\frac{1}{r^{2} \sin ^{2} \theta}\right] \boldsymbol{u}
$$

where $\nu_{T}$ is a turbulent viscosity.

The radially truncated model equations have been solved numerically with an explicit time stepping method of DuFort-Frankel type with suitable boundary conditions. For full details we address the reader to the extensive paper by Belvedere, Pidatella and Proctor (1990) who calculated a similar model in the solar convection zone.

Assuming the differential rotation profile $u_{0}$ in the boundary layer as given by interpolating the most recent helioseismological data, the results show the existence of periodic (dynamo wave-like) stable solutions with both equatorward and poleward migrating branches, as shown by the associated butterfly diagram (Fig. 1).

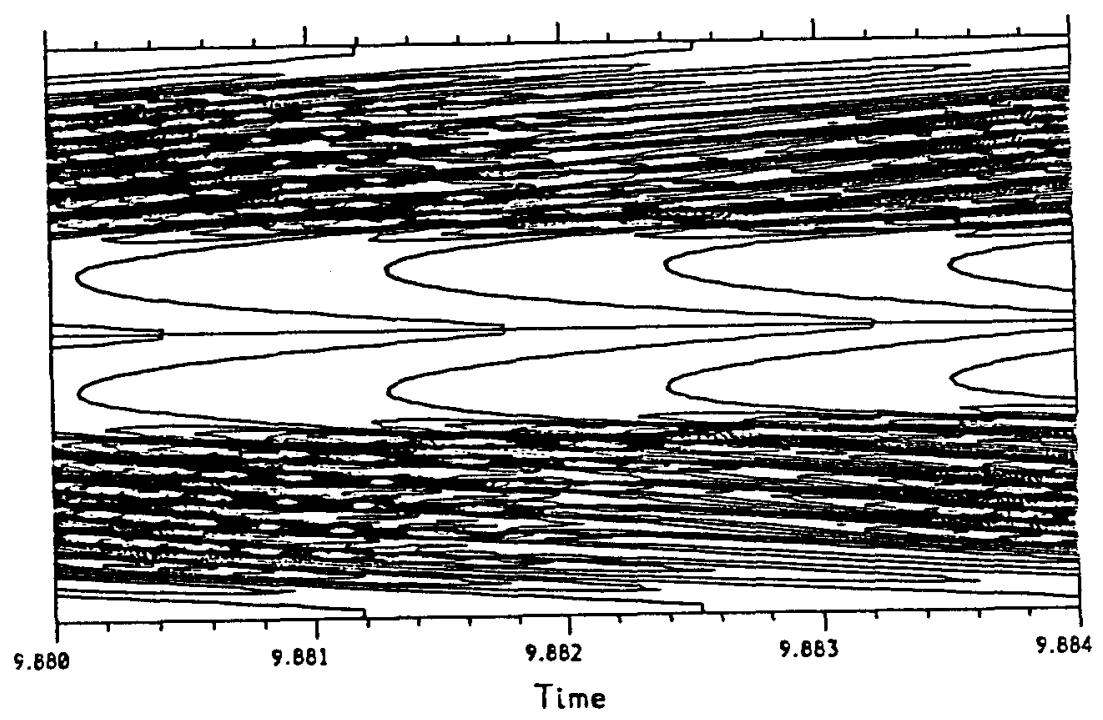

Fig. 1. Butterfly diagram for $D=-1$ and $d=0.05 R_{\odot}$

These solutions are found for dynamo number $D=\alpha \omega_{\circ} d^{3} / \eta_{T}^{2} \approx-1$, where $d$ is the thickness of the boundary layer and $\omega_{0}$ is the surface angular velocity at latitude of $\lambda=37^{\circ}$. Incidentally, the relatively small value of $|D|$ is a consequence of the very steep radial angular velocity gradient in the thin layer, since for the onset of dynamo action $|\alpha \partial \omega / \partial r|$ must exceed a characteristic value.

The relative latitude extent and magnetic field intensity of the polar and equatorial branches shown in Fig. 1 is somewhat artificial, as it depends on the free choice of the latitudinal variation of the model parameter $\alpha$, which has only to satisfy the requirement of antisymmetry with respect to the equator. Here we adopted $\alpha(\theta) \approx \sin \theta \cos \theta$, where $\theta$ is the polar angle, this giving more emphasis to the polar branch. 


\section{Conclusions}

The basic result of our numerical simulation is that horizontally propagating $\mathrm{dy}$ namo waves, with equatorial and polar branches, can exist even in a very thin layer, such as the boundary layer at the bottom of the convection zone, where the radial angular velocity gradient changes its sign at latitude $\lambda=37^{\circ}$.

Therefore this gives support to our idea that the observed latitude distribution of solar activity belts is a natural consequence of the profile of angular velocity inside the Sun, namely of the fact that the rigid rotation angular velocity beneath the convection zone has approximately the same value as the surface (and c.z.) angular velocity at latitude $\lambda=37^{\circ}$.

Of course it remains to be understood why the internal rotation is rigid and its angular velocity coincides with that at the surface just at latitude $\lambda=37^{\circ}$; and why do spots occur only for $\lambda<35^{\circ}-40^{\circ}$. A great amount of detailed study of the interaction of rotation, convection and magnetic field in the solar interior is clearly needed to try to explain these facts.

Nevertheless the present framework allows us to reasonably suggest that observation of latitude distribution and migration of stellar active regions on late main sequence $(\mathrm{G}, \mathrm{K}, \mathrm{M})$ slowly rotating stars, by the available sophisticated photometric and spectroscopic methods, may conversely give us the possibility to infer their internal rotation profile and angular momentum distribution in a simple and direct way, offering a powerful tool to investigate stellar internal rotation.

Any results obtained by a methodology based on the principles outlined here, should be compared with future stellar oscillations data from space, presumably in the next decade, in order to test the validity of the boundary layer dynamo hypothesis on a large sample of objects.

\section{References}

Belvedere, G:: 1990, in Inside the Sun, Proc. IAU Coll. 121, G. Berthomieu and M. Cribier eds., Kluwer, Dordrecht, p. 371

Belvedere, G., Pidatella, R.M., Proctor, M.R.E.: 1990, Geophys. Astrophys. Fluid Dyn. 51,263

Brown, T.M., Christensen-Dalsgaard, J., Dziembowski, W., Goode, P.R., Gough, D.O., Morrow, C.A.: 1989, Astrophys. J. 343, 526

Dziembowski, W.A., Goode, P.R., Libbrecht K.G.: 1989, Astrophys. J. 337, L53

Glatzmaier, G.A.: 1985a, Geophys. Astrophys. Fluid Dyn. 31, 137

Glatzmaier, G.A.: 1985b, Astrophys. J. 291, 300

Harvey, J.W.: 1988, in Seismology of the Sun and Sun-like Stars, E.J. Rolfe ed., ESA-SP 286, 55

Libbrecht, K.G.: 1989, Astrophys. J. 336, 1092

Parker, E.N.: 1955, Astrophys. J. 122, 293

Yoshimura, H.: 1975a, Astrophys. J. Suppl. 29, 467

Yoshimura, H.: 1975b, Astrophys. J. 201, 740 